\title{
Laserterapia de baixa potência no tratamento da hipersensibilidade dentinária
}

\author{
Low-level laser therapy in the treatment of dentin hypersensitivity
}

Ricardo Sartori*

Priscila Portella Soares ${ }^{* *}$

\section{Resumo}

Objetivos: avaliar, por meio de métodos clínicos, a eficácia da laserterapia de baixa potência no tratamento da hipersensibilidade dentinária, orientar os tipos de tratamento aos pacientes e proporcionar melhor qualidade de vida, interferindo diretamente no bem-estar diário, uma vez que a sensibilidade se mostra como uma das complicações mais dolorosas e resistentes. Métodos: foram selecionados 72 dentes de 23 pacientes sob dois testes: táctil e térmico evaporativo. Os dentes foram posteriormente divididos aleatoriamente em dois grupos: o grupo placebo (36 dentes) e o grupo laser (36 dentes), submetidos à terapia com laser de baixa potência, em que foram realizadas quatro sessões de aplicações, uma vez por semana. Resultados: o grupo laser apresentou diferença estatisticamente significante ( $p>0,05)$ na redução da sensibilidade dolorosa quando comparado ao grupo placebo tanto no teste táctil como no teste térmico evaporativo. Conclusão: a terapia com laser de baixa potência é eficaz e mostra-se muito promissora para o tratamento da hipersensibilidade dentinária.

Palavras-chave: Hipersensibilidade dentinária. Laserterapia de baixa potência. Laserterapia no tratamento da hipersensibilidade dentinária.

\section{Introdução}

Sabe-se que a hipersensibilidade dentinária é um desconforto muito comum, que gera grande incômodo no dia a dia das pessoas, levando-as a buscar tratamento que seja capaz de solucioná-la. Apesar de serem testados diversos tipos de dessensibilizantes, não há no mercado uma técnica que seja totalmente eficaz, ainda que $35 \%$ da população mundial sofra com esse problema ${ }^{1}$.

De acordo com Walters ${ }^{2}$ (2005), várias teorias são citadas para explicar o mecanismo envolvido na hipersensibilidade dentinária. Atualmente, a Teoria Hidrodinâmica de Brannstrom é a mais aceita, baseada em que os túbulos dentinários estejam preenchidos por um fluido que, ao sofrer um estímulo, produz a movimentação desse líquido, que acaba atingindo as terminações nervosas em toda a extensão dos túbulos, gerando a dor ${ }^{3}$.

Pereira $^{4}$ (1995) descreve que a sensibilidade é provocada por estímulos não nocivos, sem ligação com patologias pulpares, pois, quando sobrepostos à dentina, geram dor sem provocar alterações patológicas. De acordo com Pashley et al. ${ }^{5}$ (2008), quando há exposição dos túbulos dentinários na região cer- 
vical dos dentes, acredita-se que pode ser em função dos processos de erosão, abfração ou abrasão, desgastes que dizem respeito à perda da estrutura dental. Sobral e Garone Neto ${ }^{6}$ (1999) salientam a importância de identificar as lesões não cariosas que expõem a região cervical e classificam: abrasão como desgaste mecânico da estrutura dentária pela constante fricção de um corpo estranho; erosão como perda superficial dos tecidos duros dentários gerados por processos químicos não contendo bactérias; abfração como lesões que surgem pela presença de traumatismo/interferências oclusais, força mastigatória ou do apertamento dentário, levando à deflexão da estrutura dentária.

A hipersensibilidade dentinária é reconhecida como dor aguda, de curta duração, resultante da exposição da dentina em resposta aos estímulos térmicos, evaporativos, tácteis, osmóticos ou quími$\cos ^{7-9}$. A relação entre a aplicação do laser de baixa potência e o possível alívio da dor tem acumulado, nas últimas décadas, pesquisas com resultados positivos devido à sua alta taxa de sucesso na diminuição do incômodo causado pela exposição dos túbulos dentinários na cavidade oral ${ }^{10}$.

A sensibilidade deve ser tratada a fim de oferecer aos pacientes o conforto oral diário e a melhora na qualidade de vida, independentemente de sua etiologia. Conforme Midda e Renton-Harper ${ }^{11}$ (1991), os lasers de baixa potência atuam com pequenos comprimentos de onda e produzem pouco aumento da temperatura. Esses comprimentos de onda são suficientes para estimular a circulação e a atividade celular pelo aumento da produção de ATP mitocondrial, estimulando terminações nervosas, resultando em ação analgésica.

Dessa forma, este trabalho teve como principais objetivos: avaliar, por meio de métodos clínicos, a eficácia da laserterapia de baixa potência no tratamento da hipersensibilidade dentinária, orientar os tipos de tratamento aos pacientes e proporcionar melhor qualidade de vida.

\section{Métodos}

A presente pesquisa caracteriza-se como um estudo experimental, do tipo exposto-controle, com o objetivo de verificar, por intermédio de métodos clínicos, a eficácia da laserterapia de baixa potência no tratamento da hipersensibilidade dentinária. O estudo foi aprovado pelo Comitê de Ética em Pesquisa da Universidade de Santa Cruz do Sul (Unisc) sob parecer número 2.078.092.

A amostra foi constituída por 72 dentes de 23 pacientes de ambos os sexos, selecionados na disciplina Periodontia, na Clínica de Odontologia da Unisc, que apresentaram sintomatologia dolorosa nos testes táctil (estímulo mecânico gerado pela sonda exploradora $\mathrm{n}^{\mathrm{0}} 5$ ) e térmico-evaporativo (jato de ar com a seringa tríplice em temperatura am- biente a aproximadamente $1 \mathrm{~mm}$ de distância), ambos realizados perpendicularmente na face vestibular e lingual dos dentes, durante 5 segundos.

Como critérios de inclusão, foram eleitos os dentes que apresentavam sensibilidade no teste táctil ou no térmico-evaporativo. Os fatores considerados como critérios de exclusão foram: pacientes sob tratamento com analgésico, anti-inflamatório ou qualquer outra medicação que pudesse mascarar a sintomatologia dolorosa, dentes submetidos a tratamento periodontal cirúrgico há menos de dois meses, dentes com sinais e sintomas de problemas pulpares, presença de tecido cariado, restaurações, tratamento endodôntico, trincas, fraturas, utilização de aparelhos fixos ou dentes com mobilidade.

Conforme Martineli et al. ${ }^{12}$ (2001), para validação das respostas aos estímulos, foi utilizada uma escala de medida verbal (EMV), uma vez que os pacientes apresentam dificuldade em expressar e interpretar a intensidade da dor. A EMV apresenta quatro níveis de dor, expressos em números de zero a três: zero para dor ausente ou nenhum desconforto; um para dor mínima ou mínimo desconforto; dois para dor moderada ou médio desconforto; e três para dor intensa ou grande desconforto por mais de 10 segundos (Quadro 1).

Quadro 1 - Escala de medida verbal

\begin{tabular}{|l|}
\hline Escala de medida verbal \\
$0=$ dor ausente ou nenhum desconforto \\
$1=$ dor mínima ou mínimo desconforto \\
$2=$ dor moderada ou médio desconforto \\
$3=$ dor intensa ou grande desconforto por mais de 10 segundos \\
Fonte: Martineli et al. ${ }^{2}$ (2001).
\end{tabular}

O grupo laser foi submetido às aplicações com laser de baixa potência, realizadas uma vez por semana durante quatro semanas, de maneira pontual, o mais perpendicular possível ao dente, em quatro pontos na região cervical (mesial, médio e distal da face vestibular e um ponto fixo na face palatina/lingual). O grupo placebo foi usado para descartar o efeito da sugestão na melhora dos pacientes testados. Foi utilizado o aparelho fotopolimerizador, que emite luz azul e é utilizado em restaurações de resina fotoativada, para polimerizá-la. A luz foi aplicada uma vez por semana durante quatro semanas, posicionada em três pontos (vestibular, oclusal e palatino/lingual). A umidade da cavidade oral foi controlada com roletes de algodão e sugador descartável, por meio do isolamento relativo dos dentes a serem tratados, uma vez que o procedimento era de curta duração.

O laser terapêutico utilizado foi o Therapy XT, à base de diodo, cujo meio ativo é composto por $\mathrm{HeNe}$ (hélio-neônio) e GaAlAs (arseneto de gálio-alumínio), que possuem um comprimento de onda entre $630 \mathrm{~nm}$ e $810 \mathrm{~nm}$. A dose aplicada foi selecionada de acordo com a relação da potência do aparelho 
(100 $\mathrm{mW}$ ) versus tempo (segundos), resultando em $1 \mathrm{~J}$ aplicado a cada 10 segundos, ou seja, 40 segundos em cada dente por sessão de aplicação. A dosimetria ideal para obtenção de resultados é fundamental, doses muito baixas (abaixo de $1 \mathrm{~J} / \mathrm{cm}^{2}$ ) não promovem bioestimulação e doses exageradas (acima de $5 \mathrm{~J} / \mathrm{cm}^{2}$ ) promovem interdição nos processos metabólicos ${ }^{13}$. Sendo assim, a dosimetria total aplicada em cada dente foi de $4 \mathrm{~J} / \mathrm{cm}^{2}$.

\section{Resultados}

Os resultados foram tratados estatisticamente por meio de análise de variância (ANOVA) de acordo com sua distribuição. Para os dados paramétricos, foi utilizada a análise de variância de Bonferroni e, para os dados não paramétricos, a análise de variância de Kruskal-Wallis, ajustados pelo teste de Dunn. Ambos os dados foram analisados com intervalo de confiança de $5 \%(\mathrm{p}<0,05)$.
O grupo laser, avaliado sob estímulo tanto térmico (T1) quanto táctil (T2), apresentou diferença estatisticamente significante quando comparado no geral (G) de quatro sessões, já o grupo placebo, quando avaliado sob as mesmas condições, não diferiu estatisticamente (Tabela 1). Quando são avaliados os dois grupos sob as condições iniciais do tratamento ( $1^{\underline{a}}$ sessão) e após a última aplicação ( $4^{\mathrm{a}}$ sessão), nos testes térmico (T1) e táctil (T2), nota-se significativamente a redução da sensibilidade dolorosa no grupo laser (Tabela 2 ).

Tabela 1 - Médias dos níveis de dor referentes à EMV, comparados ambos os grupos entre as quatro sessões, Santa Cruz do Sul, 2017

\begin{tabular}{l|r|r|r|c}
\hline \multicolumn{1}{c|}{ Grupo } & $\mathrm{n}$ & $1^{\mathrm{a}}$ sessão $(\mathrm{G})$ & $4^{\mathrm{a}} \operatorname{sessão}(\mathrm{G})$ & $\mathrm{p}$ \\
\hline Laser & 36 & 1,31 & 0,18 & $<0,0001$ \\
Placebo & 36 & 0,90 & 0,79 & $<0,2697$ \\
\hline
\end{tabular}

Fonte: elaboração dos autores.

Tabela 2 - Médias dos níveis de dor referentes à EMV, entre ambos os grupos sob os estímulos térmico (T1) e táctil (T2) entre a $1^{\underline{a}}$ e a $4^{a}$ sessões, Santa Cruz do Sul, 2017

\begin{tabular}{|c|c|c|c|c|c|c|c|}
\hline Grupo & $n$ & $1^{\mathrm{a}}$ sessão $(\mathrm{T} 1)$ & $4^{\mathrm{a}}$ sessão $(\mathrm{T} 1)$ & $\mathrm{p}$ & $1^{\mathrm{a}}$ sessão (T2) & $4^{\mathrm{a}}$ sessão (T2) & $\mathrm{p}$ \\
\hline Laser & 36 & 2,00 & 0,28 & $<0,0001$ & 0,61 & 0,08 & $<0,0245$ \\
\hline Placebo & 36 & 1,47 & 1,31 & $<0,2827$ & 0,33 & 0,28 & $<0,6042$ \\
\hline
\end{tabular}

Fonte: elaboração dos autores.

Avaliando-se os níveis de dor (EMV) nos grupos placebo e laser, quando comparados entre a condição inicial ( $1^{\underline{a}}$ sessão) e após a última aplicação ( $4^{\mathrm{a}}$ sessão), somente sob o estímulo térmico (T1), há uma diferença estatística significante na redução da dor no grupo laser. Quando observados os níveis de dor (EMV) entre a condição inicial ( $1^{\mathrm{a}}$ sessão) e após a última aplicação ( $4^{\mathrm{a}}$ sessão), somente sob o estímulo táctil (T2), o grupo laser também apresenta resultado significante na redução da dor. No grupo placebo, apesar da redução numérica nos níveis de dor, estatisticamente, os valores são insignificantes (Tabela 2).

Quando observamos os grupos somente entre as primeiras sessões, nota-se que, no geral, o teste térmico (T1) obteve uma média do nível de dor muito superior se comparado ao teste táctil (T2). Ao final das quatro sessões, nota-se que o grupo laser obteve um resultado eficaz, ou seja, estatisticamente significante na redução da dor sob ambos os estímulos (Tabela 3).

Tabela 3 - Médias dos níveis de dor referentes à EMV quando avaliados os grupos laser e placebo separadamente, sob os testes T1 e T2 (1 ${ }^{a}$ sessões) e T1 e T2 (4ª sessões), Santa Cruz do Sul, 2017

\begin{tabular}{|c|c|c|c|c|c|c|}
\hline $\begin{array}{c}\text { Teste/ } \\
\text { avaliação }\end{array}$ & $\mathrm{n}$ & $\begin{array}{c}1 \text { a } \\
\text { sessão } \\
\text { Laser }\end{array}$ & $\begin{array}{c}1 \text { a } \\
\text { sessão } \\
\text { Placebo }\end{array}$ & $\begin{array}{c}4^{\mathrm{a}} \\
\text { sessão } \\
\text { Laser }\end{array}$ & $\begin{array}{c}4^{\underline{a}} \\
\text { sessão } \\
\text { Placebo }\end{array}$ & p \\
\hline Térmico (T1) & 72 & 2,00 & 1,47 & 0,28 & 1,31 & $<0,0001$ \\
\hline Táctil (T2) & 72 & 0,61 & 0,33 & 0,08 & 0,28 & $<0,5282$ \\
\hline
\end{tabular}

Fonte: elaboração dos autores.

\section{Discussão}

De acordo com Silva et al. ${ }^{14}$ (2005), o correto diagnóstico para sensibilidade é extremamente importante, uma vez que ela pode ser confundida com outras patologias dentárias de sintomas similares. Segundo Pereira ${ }^{4}$ (1995), se a dor for localizada, provocada por estímulos, aguda, passageira em curto espaço de tempo, transitória e, algumas vezes, condizer com o estado emocional do paciente, esse contexto é sugestivo de hipersensibilidade.

Grossman $^{15}$ (1935) destacou alguns requisitos importantes que determinam o tratamento ideal da sensibilidade que devem ser seguidos até hoje, como: ser biocompatível, agir rapidamente, ser eficaz por longos períodos, ter fácil aplicação, não lesionar a polpa, não gerar dor ou desconforto e não alterar a cor dos dentes.

Conforme $\operatorname{Sobral}^{16}$ (2003), Aguiar et al. ${ }^{17}$ (2005), Conceição ${ }^{18}$ (2007) e Shintome et al. ${ }^{19}$ (2007), a hipersensibilidade pode ser resolvida com dentifrícios, flúor, dessensibilizantes, adesivos dentários, laserterapia, restaurações, cirurgias mucogengivais e tratamento endodôntico. No entanto, Ladalardo et al. $^{20}$ (2004), em seu estudo, afirmam que a literatura é unânime em demonstrar que, mesmo com uma diversidade de opções para o tratamento da hipersensibilidade dentinária, ainda não se encontra tratamento que reduza a dor a níveis satisfatórios. 
Embora o mecanismo de ação do laser de baixa potência ainda não se apresente totalmente conhecido, acredita-se que a irradiação sob a dentina exposta estimule as células nervosas do tecido pulpar, interferindo na polaridade das membranas celulares, levando ao bloqueio do estímulo nervoso ${ }^{21}$. Sendo assim, conforme Silva et al..$^{22}$ (2010) e Lins et al. ${ }^{23}$ (2013), os lasers de baixa potência produzem efeito analgésico devido à queda da transmissão nervosa e, além disso, causam aceleração no processo cicatricial, devido ao aumento da proliferação celular.

Neste estudo, observou-se que a utilização de laser de baixa potência no tratamento da hipersensibilidade dentinária, avaliado tanto sob o estímulo térmico evaporativo (T1) quanto sob o estímulo táctil (T2), apresentou diferença estatisticamente significante na redução dos estímulos dolorosos entre a condição inicial do tratamento ( $1^{\mathrm{a}}$ sessão) e após

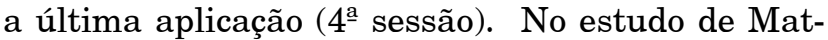
sumoto et al. ${ }^{24}$ (1986), a terapia com laser de baixa potência apresentou resultados positivos somente em pacientes que, inicialmente, apresentaram dor nos níveis leve ou moderado.

Análises demonstram que os lasers de baixa potência podem reduzir de $65 \%$ a $75 \%$ a hipersensibilidade, sem danos pulpares, mantendo-se por até seis meses, período de observação do estudo de Ciaramicoli et al..$^{25}$ (2003). Pinheiro et al. ${ }^{26}$ (1998) perceberam que sessões isoladas de laserterapia não são eficazes para promover a dessensibilização a estímulos gerados por ar, mas, no entanto, duas ou três sessões com intervalos de quatro dias demonstraram procedimentos bem-sucedidos, gerando, assim, concordância com o estudo de Marsílio et al. ${ }^{27}$ (2003), o qual concluiu que, para reduzir a dor dentinária, deve-se ter um intervalo de tempo de, no mínimo, 72 horas até a próxima irradiação.

Nesta pesquisa, as aplicações foram realizadas uma vez por semana durante quatro semanas, e os resultados foram estatisticamente significantes quando comparados entre a primeira e última sessão tanto no teste térmico evaporativo (T1) quanto no táctil (T2).

Comparando os resultados entre os grupos laser e placebo sob ambos os estímulos, nota-se um resultado extremamente significante na redução da sensibilidade dolorosa no grupo laser, porém, apesar de não diferir estatisticamente, o grupo placebo também apresenta melhoria na redução da dor. De acordo com Dantas et al. ${ }^{28}$ (2013), esse fato deve ser levado em consideração, já que, em alguns casos, o placebo também demonstra resultados próximos aos do grupo tratado, necessitando, talvez, de controles por períodos mais prolongados, para que, então, o efeito placebo possa ser eliminado e, com isso, se confirme com maior certeza o efeito real do laser.

\section{Considerações finais}

A sensibilidade dentinária é um contratempo comum nas atividades clínicas no dia a dia do cirurgião-dentista. Quando um paciente apresenta sintomas, deve-se, inicialmente, realizar o diagnóstico correto, informá-lo sobre as diversas opções de tratamento para eliminar o problema e conscientizá-lo de que o alívio da dor terá maior sucesso se combinado com a remoção do estímulo nocivo causador. No entanto, a terapia com laser de baixa potência foi eficaz neste estudo, mostrando-se promissora para o tratamento da hipersensibilidade dentinária, visto que o grupo laser apresentou diferença estatisticamente significante ( $>0,05)$ na redução da sensibilidade dolorosa quando comparado ao grupo placebo, tanto no teste táctil como no teste térmico evaporativo.

\section{Abstract}

Objective: to assess, through clinical methods the efficacy of low-level laser therapy on the treatment of dentin hypersensitivity, to instruct patients on the types of treatment, and to provide an improved quality of life, thus affecting directly daily well-being considering that sensitivity is one of the most painful and resistant complications. Methods: a total of 72 teeth were selected from 23 patients by means of two tests: tactical and thermal evaporation. The teeth were later divided randomly into two groups: a placebo group (36 teeth) and a laser group (36 teeth), which were subjected to four sessions of low-level laser therapy once a week. Results: the laser group has presented significant statistical difference $(p>0.05)$ in the reduction of painful sensitivity when compared to the placebo group, in both the tactical and thermal evaporation tests. Conclusion: the low-level laser therapy was effective and it has been shown as a promising treatment for dentin hypersensitivity.

Keywords: Dentin hypersensitivity. Low-level laser therapy. Laser therapy in the treatment of dentin hypersensitivity.

\section{Referências}

1. Almeida ECB, Menezes MRM, Aguiar CM. Tratamento da Hiperestesia dentinária com laser de GaAIAs. Odontol Clín Científ 2006; 5(2):143-15.

2. Walters PA. Dentinal hypersensitivity: A Review. J Contemp Dent Pract 2005; 6(2):107-117.

3. Brannstrom M. The hydrodynamic theory of dentinal pain: sensation in preparations, caries and the dentinal crack. J Endod 1984; 42(6):331-333.

4. Pereira JC. Hiperestesia dentinária - aspectos clínicos e formas de tratamento. MaxiOdonto 1995; 1(2):1-24.

5. Pashley DH, Tay FR, Haywood VB, Collins MA, Drisko CL. Dentin hypersensitivity: consensus-based recommendations for the diagnosis and management of dentin hypersensitivity. Inside Dent 2008; 4(9):1-9.

6. Sobral MAP, Garone Neto N. Aspectos clínicos da etiologia da hipersensibilidade dentinária cervical. Rev Odontol Univ 1999; 13(2):189-95. 
7. Garone Filho GW. Lesões cervicais e hipersensibilidade dentinária. In: Todescan FF, Bottino MA. Atualização clínica odontológica. São Paulo: Artes Médicas; 1996. p. 35-73.

8. Cummins D. Dentin Hypersensitivity: From Diagnosis to a breakthrough therapy for everyday sensitivity refief. J Clin Den 2009; 20(1):1-9.

9. Dantas EM, Dantas PMC, Nóbrega FJO, Vasconcelos RG, Júnior JNA, Queiroz LMG. Tratamento da hipersensibilidade dentinária cervical com laser de baixa potência - revisão de literatura. Odontol Clín Cient 2013; 12(1):7-11.

10. Costa LM, Cury MS, Oliveira MAHM, Nogueira RD., Geraldo-Martins VRA. Utilização da Laserterapia para o Tratamento da Hipersensibilidade Dentinária: Revisão de Literatura. 2016; 18(3):210-6.

11. Midda M, Renton-Rarper P. Laser in Dentistry. Br Dent J 1991; 170(9):343-6.

12. Martineli ACBF, Santiago SL, Pereira JC. Avaliação da eficácia de agentes antihiperestésicos: métodos clínicos e laboratoriais. Rev FOB 2001; 9(3/4):157-166.

13. Basting RT, Silveira AP, Batista IO. Tratamento da hipersensibilidade dentinária com laser de baixa intensidade. Arq em Odontol 2008; 44(2):88-92.

14. Silva CHV da, Souza FB de, Guimarães RP, Lyra AMV de C, Loretto SC, Braz R, Cabral AJ, Beatrice LC de S, Correia M das N. Diagnóstico e Plano de Tratamento. In: Busato ALS. Dentística: filosofia, conceitos e prática clínica - Grupo Brasileiro de Professores de Dentística. São Paulo: Artes Médicas; 2005. p. 95-124.

15. Grossman LA. Systematic method for the treatment of hypersensitivity dentine. J Am Dent Assoc 1935; 22(4):592-598.

16. Sobral MAP. Lesões cervicais não cariosas e hipersensibilidade dentinária cervical. In: Garone Netto N. Introdução a Dentística Restauradora. São Paulo: Santos; 2003. p. 265-283.

17. Aguiar FHB, Giovanni EM, Monteiro FHL, Villalba H, Melo JJ de, Tortamano N. Hipersensibilidade Dentinária - causas e tratamento. Uma revisão de literatura. Rev Inst Ciênc Saúde 2005; 23(1):67-71.

18. Conceição EN. Dentística: saúde e estética. Porto Alegre: Artes médicas; 2007, $2^{\mathrm{a}}$ ed.

19. Shintome LK, Umetsubo LS, Nagayasso MP, Jorge ALC, Gonçalves SE de P, Torres CRG. Avaliação Clínica da Laserterapia no Tratamento da Hipersensibilidade Dentinária. Cienc Odontol Bras 2007; 10(1):26-33.

20. Ladalardo TCCGP, Pinheiro A, Campos RA, Brugnera Júnior A, Zanin F, Albernaz PL, Weckx LL. Laser Therapy in the Treatment of Dentine Hypersensitivity. Braz Dent J 2004; 15(2):144-150.

21. Pesevska S, Nakova M, Ivanovski K, Angelov N, Kesic L, Obradovic R, Mindova S. Dentinal hypersensitivity following scaling and root planning: comparison of lowlevel laser and topical fluoride treatment. Lasers Med Sci 2010; 25(5):647-50.

22. Silva JP da, Silva MA da, Almeida AP, Lombardi Júnior I, Matos AP. Laser therapy in the tissue repair process: a literature review. Photomed Laser Surg 2010; 28(1):17-21.

23. Lins EC, Oliveira CF, Guimarães OC, Costa CA, Kurachi C, Bagnato VS. A novel 785-nm laser diode-based system for standardization of cell culture irradiation. Photomed Laser Surg 2013; 31(10):466-73

24. Matsumoto K, Nakamura G, Tomonari H. Study on the treatment of hypersensitivity dentine By HeNe Laser irradiation. J Conservat Dent 1986; 29:312-317.
25. Ciaramicoli MT, Carvalho RC, Eduardo CP. Treatment of cerical dentin hypersensitivity using neodyum: Yttrium-aluminium-garnet laser. Clin Evol Lasers Surg Med 2003; 33(5):358-62.

26. Pinheiro ALB, Brugnera Jr. A, Zanin F. Laser no tratamento da sensibilidade dentinária. In: Lasers na odontologia moderna. São Paulo: Pancast; 1998. p. 297-306.

27. Marsílio AL, Rodrigues JR, Borges AB. Effect of the clinical application of the GaAlAs laser in the treatment of dentine hypersensitivity. J Clin Laser Med Surg 2003; 21(5):291-6.

28. Dantas EM, Dantas PMC, Nóbrega FJO, Vasconcelos RG, Júnior JNA, Queiroz LMG. Tratamento da Hipersensibilidade dentinária cervical com laser de baixa potência - revisão de literatura. Odontol Clín Cient 2013; 12(1):7-11.

Endereço para correspondência:

Priscila Portella Soares

Rua Galileu Galilei, 482, Bairro Renascença

96816-862, Santa Cruz do Sul, Rio Grande do Sul, Brasil

Telefone: (53) 99903-0205

E-mail: priportellas@gmail.com

Recebido: 24/01/18. Aceito: 26/03/18. 